\title{
Design of virtual circuit experiment based on the LabVIEW
}

\author{
Hui ling Si \\ Zhengzhou university of industrial technology, Zhengzhou 451100, China \\ Xincao_123@126.com
}

Keywords: LabVIEW, Virtual circuit, Experiment.

\begin{abstract}
This article is designed by the LabVIEW circuit experiment module, common in the course each module specific program design scheme is given, and ultimately successful debugging operation. Based on the introduction of virtual instrument, can not only save costs, and to update and adjust teaching methods and means to make laboratory equipment maintain its advanced nature, improves the quality and efficiency of experiment teaching and scientific research, is a new development direction of experiment teaching, promote the laboratory technical progress.
\end{abstract}

\section{Introduction}

Obsolete equipment, backward traditional experimental teaching, has failed to keep pace with the needs of the development of education, to a great extent, restrict the development of experiment teaching and the improvement of talent training quality, moreover ordinary laboratory equipment debugging, management, easy to damage, etc.

LabVIEW programming language is flexible, open, use software to replace the function of instrument, has the advantages of good user interface, simple operation, have a virtual instrument system, and is equivalent to having a personal lab ${ }^{[1]}$.

Circuit course is electrical or related professional students learning is an important link of curriculum theory, build a virtual laboratory on the computer, using LabVIEW development platform design of virtual experiment instrument can realize the common experiment in the course, including branch current method, the first-order dynamic circuit analysis, the design of the second-order dynamic circuit analysis module, which can improve the students' learning interest, make up the lack of hardware environment experiment teaching, improving the teaching quality of circuit experiment teaching course, improve the teaching effect, can extend the students' practice platform, for the electricity class foundation courses experimental teaching to provide a new kind of auxiliary means, namely virtual instrument as the core, actual operation combined with computer simulation experiment teaching way ${ }^{[2]}$.

\section{Virtual instrument programming platform}

Virtual instrument is the development of computer hardware, software, and bus technology in the process of dense infiltration to other technology, closely combine with testing technology, instrument technology, common breed of a new product ${ }^{[3]}$. According to the journal world instrumentation and automation, the precess of this century instruments will become the development direction of electrical measuring instruments ${ }^{[4]}$.

The LabVIEW is laboratory virtual instrument engineering, Called the Laboratory Virtual Instrument Engineering Workbench(Laboratory virtual instrument integration environment ${ }^{[5]}$.It is the NI company development based on G Language (Graphics Language) of the virtual instrument development tool, is the world's first using graphical programming technology for instrument of 32-bit compiler program development system $^{[6]}$, is currently the most widely used and fastest-growing, most powerful graphical software development integration environment.

Create the core of the virtual instrument LabVIEW is VI, including Front Panel and Diagram and icon/connector ${ }^{[7]}$. 


\section{Branch current method of experimental design}

The front panel design

Through the custom controls, established the front panel of the circuit, as shown in Fig. 1. Five resistance and three power components are numeric entry control, numerical adjustable. 5 numeric display controls, used to represent five branch current.

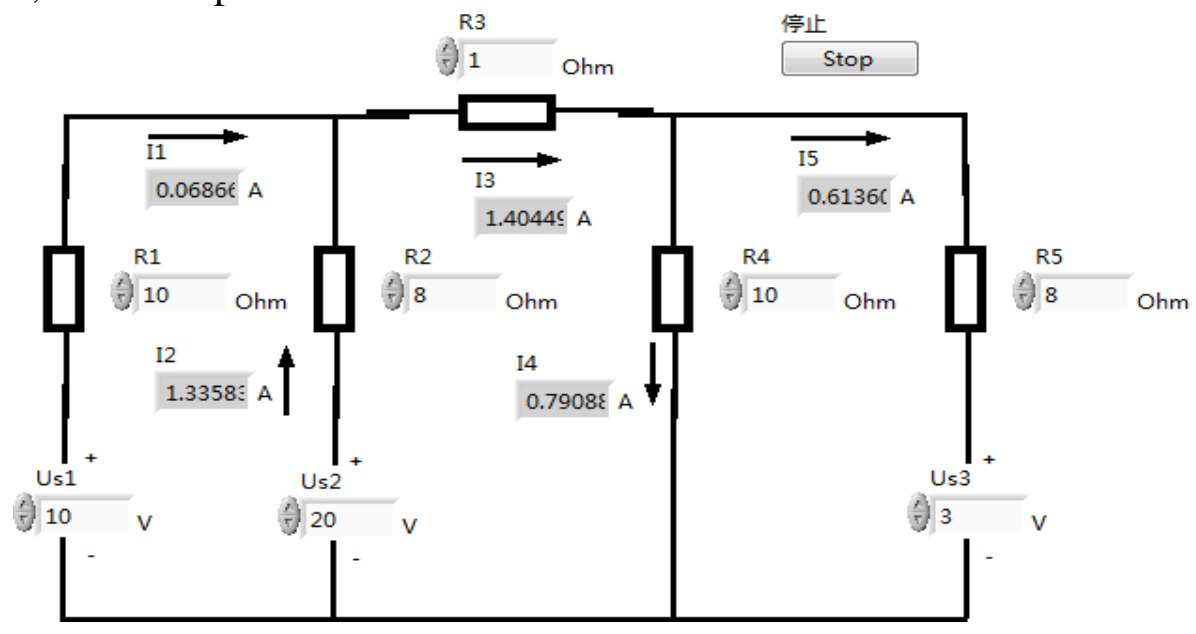

Fig. 1 The front panel of branch current method of experimental design

The diagram design

The circuit application of kirchhoff's law column to write equations again after finishing into matrix form system of linear equations.

$\left[\begin{array}{ccccc}-1 & -1 & 1 & 0 & 0 \\ 0 & 0 & -1 & 1 & 1 \\ R_{1} & -R_{2} & 0 & 0 & 0 \\ 0 & R_{2} & R_{3} & R_{4} & 0 \\ 0 & 0 & 0 & -R_{2} & R_{5}\end{array}\right]\left[\begin{array}{c}I_{1} \\ I_{2} \\ I_{3} \\ I_{4} \\ I_{5}\end{array}\right]=\left[\begin{array}{c}0 \\ 0 \\ U_{S 1}-U_{S 2} \\ U_{S 2} \\ -U_{S 3}\end{array}\right]$

Using unit conversion node to achieve conversion between units and units of data unit data, using the coefficient matrix MathScript nodes to generate linear equations and the known vector,then use Solve Linear Equations.vi to solve the linear equations.

According to the matrix equations to write programs branch current method in the analysis of circuit diagram is shown in Fig. 2.

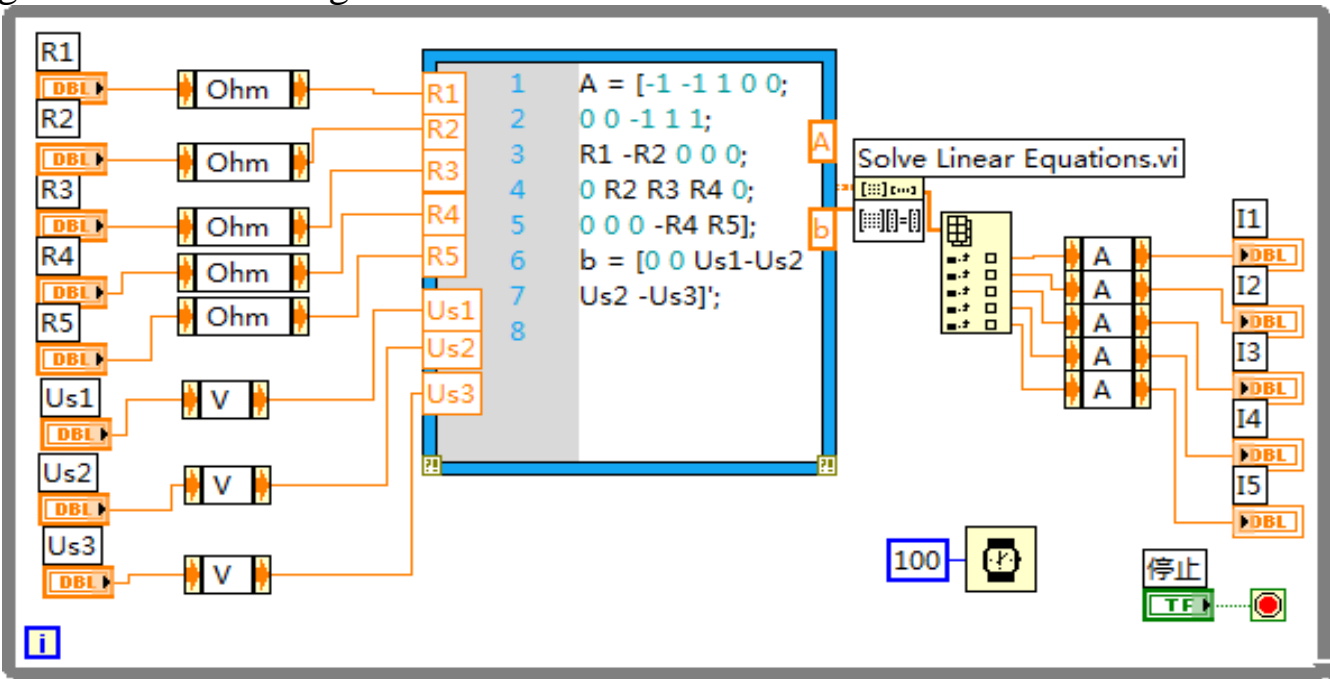

Fig. 2 The program diagram of branch current method of experimental design To run the program, the result is shown in figure 1 the front panel. 


\section{The first-order dynamic circuit design}

Dynamic change of road circuit transition from a steady state of the circuit to another steady state transition process of the dynamic circuit, the purpose of dynamic circuit analysis is to analyze the transition process of circuit works. Using LabVIEW to dynamic process simulation of the circuit, can display the parameters of the curve, helps to deepen understanding of the transition rule.

The design of the front panel

Build a circuit diagram, using XY graph to display IL, I1 and I2. The front panel is shown in Fig. 3.

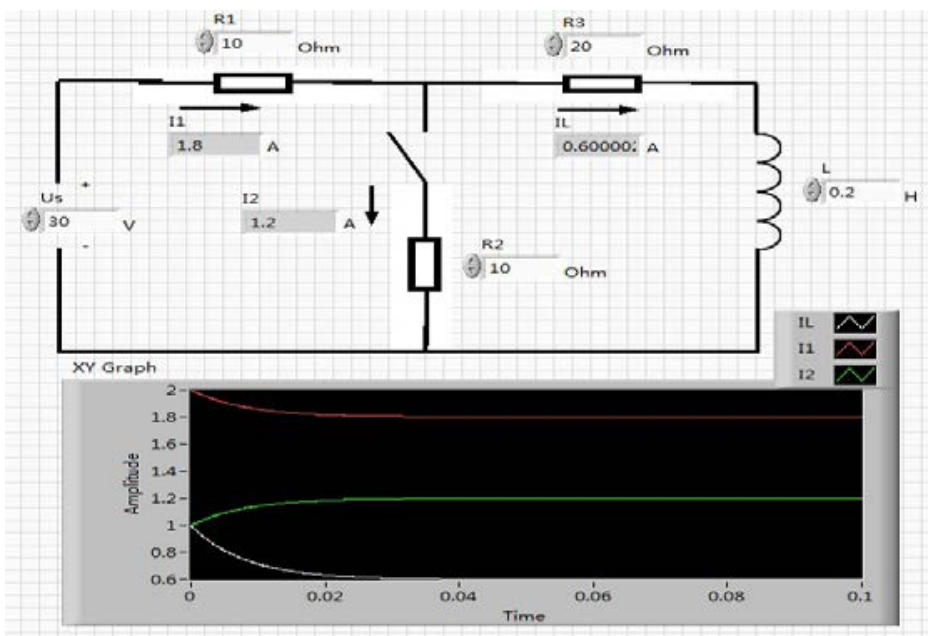

The diagram design

Fig. 3 The front panel of the first-order dynamic circuit design

According to the three key elements method to write the current expression:

$$
\begin{aligned}
& I_{l}(t)=I_{l}(\infty)+\left[I_{l}(0+)-I_{l}(\infty)\right] e^{-\frac{t}{\tau}}=\frac{3 R_{2}}{3 R_{2}+20}+\left(1-\frac{3 R_{2}}{3 R_{2}+20}\right) e^{-\frac{200+30 R_{2}}{2+0.2 R_{2}} t} \\
& I_{1}(t)=I_{1}(\infty)+\left[I_{1}(0+)-I_{1}(\infty)\right] e^{-\frac{t}{\tau}}=\frac{3 R_{2}+60}{3 R_{2}+20}+\left(\frac{30+R_{2}}{10+R_{2}}-\frac{3 R_{2}+60}{3 R_{2}+20}\right) e^{-\frac{200+30 R_{2}}{2+0.2 R_{2}} t} \\
& I_{2}(t)=I_{2}(\infty)+\left[I_{2}(0+)-I_{2}(\infty)\right] e^{-\frac{t}{\tau}}=\frac{60}{3 R_{2}+20}+\left(\frac{20}{10+R_{2}}-\frac{60}{3 R_{2}+20}\right) e^{-\frac{200+30 R_{2}}{2+0.2 R_{2}} t}
\end{aligned}
$$

According to the type (1), (2) and (3), using a formula node calculate IL, I1 and I2, program block diagram as shown in Fig. 4.

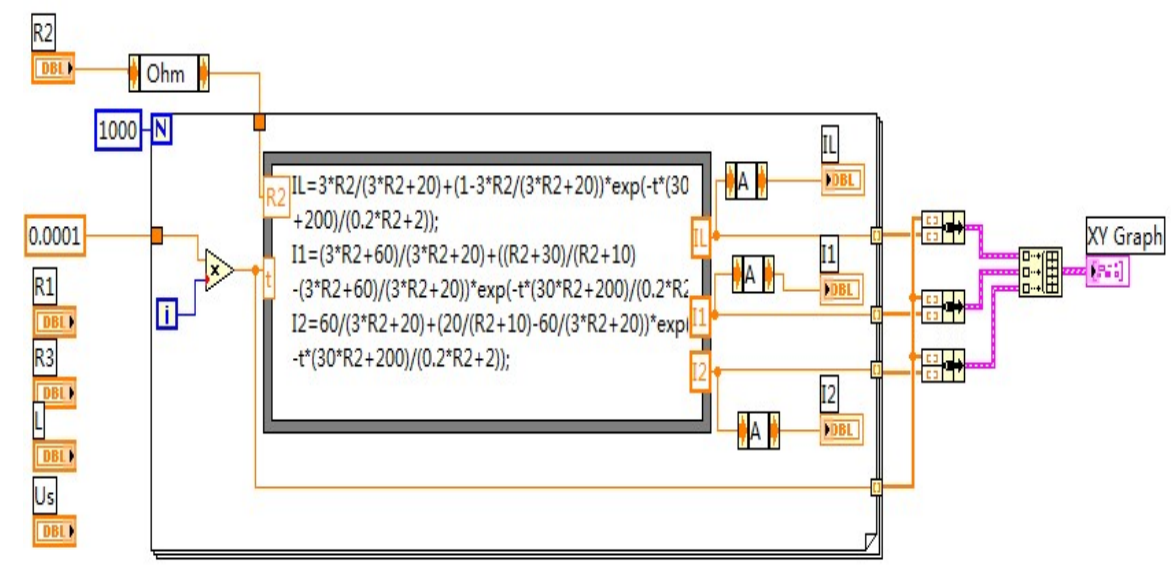

Fig.4 The first-order dynamic circuit design program block diagram 
Run results as shown in Fig. 3, in the formula node variables as R2, change the R2 value can also be used to observe the program run results.

\section{Summary}

Based on the existing conditions, using the virtual instrument technology, realize the circuit experiment design based on LabVIEW, the shortage of the experimental teaching equipment for colleges and universities to provide a new solution, further advanced experiment teaching means, experimental results more intuitive, and along with the continuous renewal of software, can make the performance and function of the experiment system more perfect, in the future the development of the laboratory has a broad application prospect.

\section{References}

[1] Hui min Yan,Wen xia Pan. Virtual instrument technique and its application in electrical experiments[J]. East China Electric Power,2002(12):56-59

[2] Ru xing Xiao, Jun long Fang .Electronic circuit virtual laboratory based on LabVIEW and

Multisim[J].Journal of Northeast Agricultural University,2008,39(4):106-108

[3] Young C P, Juang W L, Devaney M J. Real Time Intranet-Controlled Virtual Instrument Multiple-Circuit Power Monitoring. IEEE Trans IM, 2004, 49(3): 579-584

[4] C C Ko, B M Chen, S H Chen. A Large Scale Web-based Virtual Oscilloscope Laboratory Experiment, IEE Engineering Science and Education Journal,2000, 9(2): 69-76

[5] Yan hua Shen, Ru jie Wang, Zhen shan Lei. LabVIEW entry and improve the tutorial examples[M].BeiJing:China railway publishing house,2007.

[6] Chen Chun zhao, Min Zhao. The design of virtual experiment platform based on LabVIEW[J].China modern educational equipment,2009(17):31-34

[7] Qi Xie ,Xiao xing,Wen,Qin mei Gao. Design and Realization of user interface menu in LabVIEW software[J]. Micro computer information,2005,9(1): 8890. 